\title{
UN NUEVO ESTUDIO SOBRE PLATÓN
}

Franz von Kutschera, Platons Philosophie, mentis Verlag,
Paderborn, 2002, 3 vols., 749 pp.

Fernando Leal Carretero

Centro Universitario de Ciencias Sociales y Humanidades

Universidad de Guadalajara

f.leal@prodigy.net.mx

Cuando era yo estudiante de filosofía en Alemania en los años setenta, Franz von Kutschera era bien conocido por sus excelentes manuales de introducción a la lógica elemental, la lógica deóntica, la filosofía de la ciencia y, sobre todo, a la filosofía del lenguaje y a la semántica intensional. En los años ochenta y noventa, sin embargo, Kutschera comenzó a publicar obras igualmente didácticas, pero más originales y ambiciosas: una epistemología, una ética, una estética, una filosofía de la mente $y$, finalmente, un tratado sobre la filosofía en general. Pero aparte de esas obras sistemáticas, el autor ha incursionado también en la historia de la filosofía, primero con una monografía sobre Frege, y luego con un comentario sobre el Parménides de Platón, cuya enorme aportación consiste en el uso del aparato lógico de la mereología para iluminar este oscuro e intrincado diálogo. Con la extensa obra que reseño ahora, Kutschera pretende hacer una exposición de conjunto de la filosofía de Platón, diálogo por diálogo. Ésta es una tarea que tiene sus riesgos:

Debido al alud de publicaciones sobre Platón, las exposiciones de conjunto se han vuelto escasas. Sobre cada diálogo de Platón hay numerosas investigaciones y sobre cada problema particular a menudo largas discusiones. Como ya nadie puede hacerse una visión general de toda la bibliografía sobre Platón, el valor de una 
exposición de conjunto es cuestionable: corre el peligro de pasar por alto conocimientos importantes sobre problemas específicos de la interpretación de Platón. Si a pesar de ello me atrevo a hacer una exposición de conjunto, lo hago porque estoy convencido de que muchas dudas que se presentan a la hora de interpretar un diálogo desaparecen a la luz de diálogos posteriores. Los diálogos se entrecruzan temáticamente de muchas maneras, de manera que las reflexiones de Platón sobre un tema a menudo se aclaran solamente a partir de varios diálogos. ${ }^{1}$

Aparte del beneficio mencionado e indudable de una exposición de conjunto de la obra de Platón, ¿qué podríamos esperar en cuanto a resultados e interpretaciones nuevas? El propio autor habla de dos tipos de aportaciones, una sumamente importante $(\mathrm{A})$, la otra menor $(\mathrm{B})$ :

A) La mayor aportación es, sin duda, una nueva interpretación integral de las dos partes más discutidas y oscuras de la obra de Platón, el diálogo Parménides y la "doctrina no escrita":

1. En cuanto al diálogo, el libro al que aquí me refiero contiene una versión más apretada de los principales resultados de la propuesta de Kutschera, cuya exposición completa estaría en su otro libro (Platons "Parmenides"). La aportación de Kutschera consiste en mostrar que Platón hace uso de ciertos principios lógicos que pertenecen a la mereología.

2. En cuanto a la "doctrina no escrita", Kutschera pretende mostrar que su tema central es una definición lógica de los números; es decir, que Platón habría emprendido en ella el camino que después recorrería Frege. Semejante intento de Platón iría de la mano, según Kutschera, de una tendencia a matematizar el cosmos

1 "Prefacio", vol. I, p. 11. Como la paginación de cada volumen es independiente, en lo que sigue mis referencias harán uso de números romanos para cada volumen seguidos de números arábigos para las páginas. Así "(III, 43-44)" es una abreviatura de "(vol. III, pp. 43-44)". 
y, por lo tanto (a partir de la distinción entre cualidades primarias y secundarias propuesta en el Teeteto), de una devaluación de las ideas no matemáticas.

B) Aunque una exposición de conjunto permite poner en relación los distintos problemas y peculiares soluciones que constituyen la filosofía de Platón como un todo, es cierto que en el caso de muchos diálogos no es posible aportar nada particularmente nuevo. Sin embargo, Kutschera dice haber logrado una exposición mejorada de ciertos diálogos, o al menos de ciertos pasajes de algunos diálogos, que en general se consideran oscuros y se han prestado a controversias. Ésta sería una aportación menor, pero no exenta de interés de la obra objeto de este estudio. Los pasajes a los que Kutschera se refiere son:

1. La discusión sobre el saber del saber en el Carmides.

2. La teoría de los defectos en el Lisis.

3. La definición del saber como creencia verdadera fundamentada en el Teeteto.

4. La explicación del no ser en el Sofista.

El primer volumen de Platons Philosophie está dedicado a los que Kutschera sitúa dentro de la categoría de diálogos de juventud o "diálogos tempranos", considerados en el siguiente orden: Hipias Menor, Ion, Apología, Critón, Gorgias, Eutifrón, Laques, Protágoras, Lisis, Carmides, Eutidemo, Menexeno, Menón. El segundo volumen presenta los diálogos de la madurez o "diálogos intermedios", comenzando con el Fedón y siguiendo con el Simposio, la República, el Fedro, el Cratilo, el Parménides y el Teeteto. El tercero y último volumen comenta los diálogos de la vejez o "diálogos tardíos", a saber, Sofista, Político, Timeo, Critias, Filebo, Leyes. El lector informado podrá apreciar que la división en periodos que adopta Kutschera no introduce ninguna novedad, sino que utiliza la usual desde Ross. No hay ninguna referencia a las dudas surgidas más recientemente sobre la cronología o el uso de métodos estilométricos en que suele basarse. 
Aparte de los análisis de cada diálogo, el primer volumen contiene dos capítulos introductorios, uno sobre Sócrates y otro sobre la vida y los escritos de Platón; y el tercer volumen culmina con dos capítulos finales, uno dedicado a la "doctrina no escrita", y otro a una síntesis de la filosofía de Platón. Además, el tercer volumen contiene dos capítulos adicionales, con carácter de apéndices, en los que Kutschera comenta dos diálogos considerados por él apócrifos, aunque de interés para comprender la filosofía de Platón: el Hipias Mayor y el Alcibíades Mayor.

La atención y el cuidado que Kutschera invierte en los distintos diálogos no son naturalmente los mismos para todos. Los cuatro capítulos más largos están dedicados a la República, al Timeo, al Parménides y al Fedón. Los diálogos menos favorecidos son el Critón, el Laques, el Menexeno y el Critias. Entre estos dos grupos podemos distinguir grosso modo otros tres rangos de importancia, como se muestra en el cuadro de la página siguiente.

Independientemente de la extensión del comentario, cualquier autor que pretenda ofrecer una exposición general requiere algún tipo de criterio para decidir qué aspectos va a comentar. El criterio de Kutschera es "atender solamente a lo que es filosóficamente relevante" (passim). Ésta me parece una decisión perfectamente legítima: si bien hay cuestiones dramáticas o estilísticas de gran interés, incluso para los filósofos, no parece inaceptable que los zapateros atiendan ante todo a sus zapatos. Sin embargo, tal vez pudiera ser menos fácil ponerse de acuerdo entre filósofos que entre zapateros acerca de qué es y qué no es relevante para el área de trabajo correspondiente. Un libro con el título Platons Philosophie [La filosofía de Platón] no puede menos que decir, explícita o implícitamente, qué es, pues, la filosofía. Kutschera, debo decir, no es tremendamente explícito al principio del libro, pero al final del mismo ofrece una instructiva reflexión:

En su época [la de Platón], junto a la filosofía, las ciencias independientes y establecidas eran solamente la matemática, la astronomía y la medicina, y la filología apenas comenzaba. La filosofía, por tanto, era en gran medida la totalidad de las ciencias y, en 
tanto que filosofía natural, abarcaba en particular los comienzos de la física, la química, la fisiología y la biología. Con todo, Platón intentó ya delimitar el saber que atañe especialmente a la filosofía con respecto al saber especializado y repartido en diversas áreas que corresponden a las ciencias restantes. (III, 231)

\begin{tabular}{lcc}
\hline \hline Diálogo & $\begin{array}{c}\text { No. de páginas } \\
\text { edición de Stephanus }\end{array}$ & $\begin{array}{c}\text { No. de páginas } \\
\text { libro de Kutschera }\end{array}$ \\
\hline República & 294 & 57 \\
Timeo & 88 & 47 \\
Parménides & 40 & 43 \\
Fedón & 61 & 37 \\
\hline Teeteto & 68 & 31 \\
Leyes & 345 & 29 \\
Filebo & 56 & 27 \\
Gorgias & 80 & 25 \\
\hline Carmides & 23 & 21 \\
Eutidemo & 36 & 21 \\
Cratilo & 57 & 21 \\
Sofista & 52 & 21 \\
Menón & 30 & 17 \\
Simposio & 51 & 17 \\
Fedro & 52 & 17 \\
Apología & 25 & 15 \\
Protágoras & 53 & 15 \\
Lisis & 20 & 15 \\
Hipias Mayor & 23 & 15 \\
\hline Eutifrón & 14 & 11 \\
Político & 54 & 9 \\
Alcibíades & 28 & 9 \\
Hipias Menor & 13 & 7 \\
\hline Critón & 11 & 5 \\
Laques & 23 & 5 \\
Ion & 12 & 3 \\
Menexeno & 15 & 3 \\
Critias & 15 & 3 \\
\hline \hline & &
\end{tabular}


La delimitación teórica que Kutschera advierte en Platón se manifiesta en tres concepciones:

1. La filosofía sería, primero que nada, un saber del saber, que es el que permite examinar críticamente todas las pretensiones de saber que hacen los seres humanos, y merced al cual el filósofo puede purificar las almas de las falsas creencias que tienen de que saben lo que no saben (el motivo socrático del elenchos). La referencia es aquí al Carmides, y en cuanto al elenchos naturalmente a la Apología.

2. La filosofía sería, además, un saber que permite determinar el contenido de las ideas, exponer las relaciones que hay entre ellas y ordenarlas en sistemas clasificatorios de especies naturales. Este saber, llamado "dialéctica", permite ir más allá de los supuestos de las otras ciencias y fundamentarlas a partir de los primeros principios. La referencia es aquí a la República, al Parménides y al Sofista.

3. La filosofía sería, finalmente, un saber acerca del verdadero valor de las cosas, que permite ordenar y jerarquizar nuestras prioridades de acuerdo con su valor objetivo. Este saber no se opone a la "dialéctica", en tanto que Platón tiene una cosmovisión teleológica, de acuerdo con la cual el mundo está ordenado y es sólo inteligible relativamente a propiedades axiológicas reales. La referencia es aquí al Carmides y al Eutidemo (aunque en otros pasajes de la obra también al Timeo).

Dada la tercera concepción, está claro que para Platón la filosofía no es, sin embargo, un asunto de mera teoría; es evidentemente también un asunto práctico, e incluso un modo de vida. Eso no se le oculta a Kutschera, quien también ofrece una descripción en tres partes (III, 232-235). No obstante, la verdadera pasión del autor no está aquí, sino en la filosofía como teoría, y más especialmente, en la segunda concepción. Si volvemos sobre las aportaciones que Kutschera atribuye a su libro, podríamos decir que casi todas se refieren a ella (A1, A2, B3, 
B4). Se trata, sin duda, de aportaciones importantes para nuestra comprensión de la filosofía de Platón; pero la admiración que podamos sentir por ellas no debe cegarnos al hecho de que las otras dos concepciones de la filosofía en Platón se quedan un tanto cortas. Quisiera ilustrar esto con dos ejemplos, uno referido a un diálogo al que Kutschera dedica muchas páginas (el Timeo) y otro al que le dedica muy pocas (el Hipias Menor).

Comencemos con el Timeo. Nuestro autor se declara entusiasmado con el diálogo; lo llama "una obra grandiosa", en la que Platón "hace las paces con el mundo empírico" (III, 85). Todos hemos tenido enormes problemas con el texto; como dice Kutschera, "en la antiguiedad era la obra de Platón más leída y con mayor frecuencia comentada", pero "a nosotros nos cuesta mucho más trabajo" (III, 85). Son tantos los detalles que Platón nos presenta por boca de Timeo y la exposición es tan compleja y comprimida, que resulta muy difícil saber a ciencia cierta de qué está hablando Platón. Es una obra, agrega Kutschera, que no podemos entender si no tenemos siempre a la mano un buen comentario, como los de Taylor, Cornford o Vlastos. Es una pena que Kutschera, al parecer, no conozca el comentario de Brisson y Meyerstein, pues le habría interesado. En todo caso, Kutschera afirma que muchas veces ni siquiera consultar los comentarios sirve de mucho, debido a que los comentaristas se contradicen (III, 43).

¿Cuáles son, pues, los aspectos del Timeo que Kutschera entresaca por ser "filosóficamente relevantes"? Aparentemente un solo aspecto: el manejo que Platón hace de las causas.

Antes de iniciar la discusión, creo que la exposición de Kutschera es de entrada hasta cierto punto imprecisa debido a que, si bien en otros lugares de la obra advierte del peligro de interpretar a Platón a partir de Aristóteles, aquí no tiene empacho en usar la distinción aristotélica posterior entre causas formales, materiales, eficientes y finales ( $c f r$. Metafísica, Libro A). De hecho, Kutschera sugiere que es esa gran división de las causas la que dicta la organización misma del diálogo en tres partes: 1) la estructura racional del mundo (29D7-47E2), que trata de las causas formales y finales; 2) la operación de la necesidad 
(47E3-69A5), que trata de las causas eficientes; 3) la cooperación de razón y necesidad, que trata de la combinación de causas finales y eficientes (69A6-92C9). Obviamente, no habría lugar en Platón para las causas materiales de Aristóteles; y en la tercera parte del diálogo, las "causas formales ya no aparecen" (III, 44). Esto no me parece una manera adecuada de presentar las cosas. No creo que haya distinción entre causas formales y finales en Platón; o si la hay, Kutschera no explica satisfactoriamente en qué consiste. Creo también que sus causas eficientes no son distintas de las materiales: es la materia la que impone al demiurgo ciertas condiciones (las Vorbedingungen der Existenz, como las llama Kutschera) y esas condiciones constituyen justamente la causalidad eficiente. Por ello constriñen la acción teleológica, orientada al Bien, del demiurgo; a esa constricción se refiere el término de synaitía, synaítion o symmetaítion que Platón propuso en el Político en un contexto distinto, pero comparable (281C-E, 287B-D, 289C) y que retoma en el Timeo (46C-E, 76D). De hecho, esa extensión del uso del concepto de "causas auxiliares" y su aplicación a las causas eficientes, como distintas de las finales (que no serían meras causas auxiliares, sino causas en sentido propio) está en la base de la concepción fundamental de Platón, que mucho me temo se le escapa a nuestro autor.

Kutschera tiene razón al insistir en el profundo interés que tenía Platón por las matemáticas como el sustrato fundamental de la explicación del mundo; no la tiene al creer que las "causas formales" son distintas de las "causas finales". Aunque Kutschera anota que los logros de la época de Platón en materia de matemática aplicada eran más bien modestos y sus ideas algo primitivas (III, 43), Platón habría "desarrollado un gran marco teórico que parece haberse confirmado brillantemente" en la física moderna (III, 86). Con esto, Kutschera se coloca entre aquellos que consideran, al menos desde Koyré, que la batalla entre platónicos y aristotélicos en materia de física ha sido ganada finalmente por los primeros. Esto no sorprende, habida cuenta de la enorme simpatía que tiene Kutschera por la lógica, las matemáticas y la física moderna; y el poco interés que tiene por la biología, y más en general por el aristotelismo. 
(Aristóteles recibe consistentemente muy mal trato por parte de Kutschera.)

Aparte del problema de las causas, sin duda de gran interés filosófico, Kutschera lamenta que Platón no vea el llamado "problema mente-cuerpo". Nos informa (III, 58) que hay un pasaje en el Timeo donde Platón percibe el problema, pero la traducción del mismo que nos presenta en nota ("La materia participa de manera altamente inexplicable en lo espiritual", 5lA7) deja mucho que desear; por ejemplo, la palabra "materia" no aparece por ningún lado en el texto griego. En todo caso, no hay espacio aquí para discutir el asunto más por lo menudo; baste decir que Kutschera es antimaterialista y antirreduccionista, como lo muestra su tratado de filosofía de la mente (Die falsche Objektivität); y es en parte eso lo que hace que no le entusiasmen las ideas de Platón sobre el alma que parecieran poder interpretarse un poco en esa dirección. De hecho, lo único que lo entusiasma es poder hacer referencia, de vez en cuando, a teoremas matemáticos (por ejemplo, en teoría de números o de la armonía) o proposiciones físicas (por ejemplo, de óptica o de dinámica de fluidos) para aclarar ciertos pasajes. Cuando Kutschera hace esto, no hay duda que lo hace muy bien.

Curiosamente, Kutschera pretende mostrar que, si bien (como en el Fedón) se distinguen en el Timeo causas finales y causas eficientes (para usar, una vez más, la terminología aristotélica), y si bien se dice que las primeras son más importantes, mientras que las segundas son meramente "causas auxiliares", resulta que "la mayor parte de las explicaciones que Timeo da de los fenómenos son causales" (III, 44). De ahí sus enjundiosos intentos de usar teorías físicas (III, 63-64; 69-72; 78-82) para interpretar el Timeo, lo cual es muy de agradecerse. Y aunque sus explicaciones lógico-matemáticas (III, 51-57) también serán bien recibidas por sus lectores, su uso de la distinción aristotélica le impide ver que estas causas formales son justamente las causas finales, las "causas en sentido propio" (autai aitiai) que en el Político se oponen a las "causas auxiliares" de la causalidad material/eficiente (III, 44; $c f r$. III, 31). 
Me parece, pues, que Kutschera ha dado en el clavo al mostrar la articulación del Timeo en torno al problema filosófico de las causas, pero no ha penetrado todavía en el corazón del asunto: cómo las causas materiales/eficientes interactúan con las causas formales/finales. Pero no podemos esperar ni de Platón ni de un comentario a Platón que responda preguntas que todavía siguen sin respuesta en el momento actual: lo que el célebre físico Eugene Wigner llamó "the unreasonable effectiveness of mathematics in natural science" ( $c f r$. la discusión en Mark Steiner, The Applicability of Mathematics as a Philosophical Problem).

Hasta aquí, pues, lo que Kutschera hace cuando comenta el Timeo. Pero, ¿qué es lo que no hace? Kutschera declara que excluirá de su exposición todas las consideraciones médicas y fisiológicas, no poco abundantes, especialmente en la parte final del diálogo, puesto que "sólo tienen interés para la historia de la medicina" (III, 43). Con otras palabras, estas ideas no serían "filosóficamente relevantes". ¿Por qué, entonces, las ideas geométricas, armónicas, astronómicas, ópticas o dinámicas de Platón sí que son "filosóficamente relevantes", y no "sólo tienen interés para la historia de las matemáticas o de la física"? Nos quedaríamos esperando una respuesta si no tuviésemos el antecedente del ya mencionado tratado de filosofía de la mente, en que Kutschera logra hablar del problema mente-cuerpo a lo largo de 300 páginas sin mencionar ninguna investigación de las neurociencias cognitivas o de la neurofilosofía. Con esto repite la tendencia de ciertos "filósofos analíticos" de ignorar lo que estas disciplinas pueden aportar al problema. Una vez que se piensa que la aportación de esas disciplinas no es "filosóficamente relevante", resulta claro que las ideas médicas y fisiológicas de Platón tampoco lo serán.

Dicho sea de paso, algo parecido ocurre con los innumerables comentaristas de las Meditaciones de Descartes, una raza inextinguible que discurre incesantemente sobre la duda metódica, el cogito ergo sum, el argumento ontológico y el dualismo cartesiano, pero no se detiene a considerar las ideas médicas y fisiológicas con que la obra culmina y que conectan con muchos 
otros pasajes y aún obras enteras que Descartes dedicó al asunto y que eran, para él, "filosóficamente relevantes".

Pero la razón profunda que está detrás de la actitud de Kutschera (como de los comentaristas de Descartes) es cierta concepción de la filosofía contra la que, irónicamente, fue el propio Platón que advirtió, por cierto en el mismísimo Timeo, y además en un pasaje que el propio Kutschera cita. En efecto, poco antes de lamentarse que a Platón se le oculte el problema mente-cuerpo, Kutschera ha discutido el "modelo mecánico" del universo (del sistema solar, para ser exactos) y sugerido que la pregunta general de si es posible utilizar en semejante modelo solamente movimientos circulares (sistemas de epiciclos) que salven los fenómenos era conocida, si no de Platón mismo, sí del gran matemático Eudoxo, cercano a la Academia. En el Timeo, sin embargo, Platón no entra en los detalles de modelos del tipo de los de Eudoxo, sino que, dice Kutschera, "deja que Timeo diga de varias maneras que [esos detalles] son de importancia secundaria para la exposición y dan más trabajo que el tema en sentido estricto" (III, 57-58). Ese "tema en sentido estricto", das eigentliche Thema, es justamente el tema de la filosofía. ¿Y cuál es ese tema? El propio Kutschera también lo dice un poco más adelante, cuando comenta la tesis de que es un error pensar que las causas materiales y eficientes son las verdaderas causas de los fenómenos psicobiológicos, y no meras causas auxiliares de que se sirvió el dios para "llevar a cabo la idea de lo mejor según lo que es posible" (46C-D). En ese contexto, Kutschera repite con sus palabras la conclusión de Platón (III, 65). Conviene, sin embargo, atender aquí a la voz misma del filósofo griego. Léase y óigase con cuidado:

[46E] Es necesario que tratemos ambos géneros de causas por separado las que conjuntamente con la razón son artesanas de lo bello y bueno y cuantas carentes de inteligencia son origen de lo desordenado casual en todos los procesos. Ya hemos tratado, pues, las causas auxiliares adicionales de los ojos que colaboran para que alcancen la capacidad que ahora poseen. A continuación tenemos que considerar su utilidad principal, por la que dios nos los [47A] obsequió. Ciertamente, la vista, según mi entender, 
es causa de nuestro provecho más importante, porque ninguno de los discursos actuales acerca del universo hubiera sido hecho nunca si no viéramos los cuerpos celestes ni el Sol ni el cielo. En realidad, la visión del día, la noche, los meses, los periodos anuales, los equinoccios y los giros astrales no sólo dan lugar al número, sino que éstos nos dieron también la noción de tiempo y la investigación de la naturaleza del universo, de lo que [47B] nos procuramos la filosofía. Al género humano nunca llegó ni llegará un don divino mejor que éste. Por tal afirmo que éste es el mayor bien de los ojos. Y de lo restante que proveen, de menor valor, aquello que alguien no amante de la sabiduría lamentaría en vano si hubiera perdido la vista, ¿qué podríamos ensalzar? Por nuestra parte, digamos que la visión fue producida con la siguiente finalidad: dios descubrió la mirada y nos hizo un presente con ella para que la observación de las revoluciones de la inteligencia en el cielo nos permitiera aplicarlas a las de nuestro entendimiento

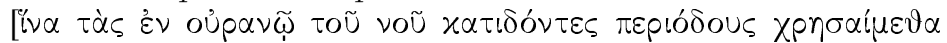

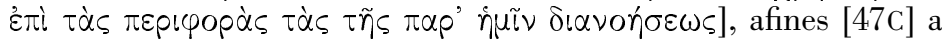
aquéllas, como pueden serlo las convulsionadas a las imperturbables, y ordenáramos nuestras revoluciones errantes por medio del aprendizaje profundo de aquéllas, de la participación en la corrección natural de su aritmética y de la imitación de las revoluciones completamente estables del dios. Y acerca de la voz y el oído, otra vez el mismo razonamiento: nos fueron concedidos por los dioses por las mismas razones y con la misma finalidad, ya que contribuye en su mayor parte a lo mismo y, a su vez, cuanto de la música [47D] utiliza la voz para ser escuchado ha sido dado por la armonía. Ésta, como tiene movimientos afines a las revoluciones que poseemos en nuestra alma, fue otorgada por las Musas al que se sirve de ellas con inteligencia, no para un placer irracional

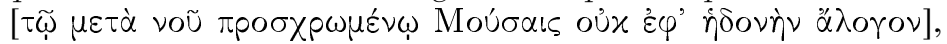
como parece ser utilizada ahora, sino como aliada para ordenar la revolución disarmónica de nuestra alma y acordarla consigo mis-

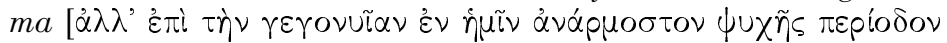

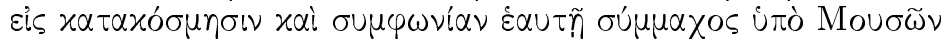
$\delta \varepsilon ́ \delta o \tau \alpha l]$. También nos ayuda en el estado sin medida y carente de gracia [47E] en el que se encuentra la mayoría de nosotros. ${ }^{2}$

2 Timeo, trad. F. Lisi; se añaden las cursivas y se introduce un pequeño cambio en la traducción. 
Como podrá apreciarse, el estudio de la astronomía, como el estudio de la música, es un medio para un fin; esto es lo que nos dice Platón. Pierre Hadot nos ha recordado de varias maneras que, para los antiguos, la filosofía es una forma de vida ( $c f r$. Qu'est-ce que la philosophie antique?). No es una ciencia en el sentido propio, si bien se vale de la ciencia para llegar adonde quiere llegar; pero, al parecer, hemos olvidado eso en el curso del último siglo.

La manera de pensar, razonar y filosofar que se presenta en el largo pasaje citado podrá sernos un poco o un mucho extraña; pero ésta es la manera platónica de hacerlo. De esto trata y no de otra cosa la filosofía de Platón. Éstas son las verdaderas causas de las cosas; y estudiarlas y meditar sobre ellas la ocupación verdadera del filósofo. Sólo mediante ellas alcanzará el filósofo la sabiduría; y alcanzando la sabiduría, alcanzará también la felicidad. No la felicidad que da el placer, el dinero, la fama o el poder. Una felicidad distinta de todas éstas es a la que el filósofo aspira; y cuyo tema es el tema de la filosofía, das eigentliche Thema, lo único que es "filosóficamente relevante".

Todas las consideraciones geométricas, armónicas, astronómicas, ópticas y dinámicas están al servicio de esa tarea. Igualmente a su servicio están todas las consideraciones médicas, fisiológicas, biológicas y psicológicas. Todas ellas son, por igual, "filosóficamente relevantes". La tarea de la filosofía las requiere todas. Cualquier intento de cercenar y poner cercos y estacas es un error. Kutschera se asombra de la enorme diversidad de intereses y conocimientos que Platón despliega en su obra (III, 174). Pues bien: ninguna de las disciplinas que Platón abraza y utiliza es idéntica a la filosofía; pero la filosofía las necesita a todas para alcanzar su propósito. Por eso es que Platón "deja que Timeo diga de varias maneras que [los detalles que competen a la ciencia] son de importancia secundaria para la exposición y dan más trabajo que el tema [de la filosofía] en sentido estricto".

Vemos, pues, que el criterio de "relevancia filosófica" que aplica Kutschera está sesgado en dirección al tipo de problemas en los que él se interesa y a los métodos que él ha utilizado para 
ocuparse de esos problemas. Pero dudo mucho que corresponda a lo que Platón hubiese considerado "relevancia filosófica". Por tanto, aunque Kutschera admite en las últimas páginas de su libro (III, 232-235) que para Platón la filosofía es un modo de vida (una Lebensform, como lo dice con terminología wittgensteiniana), no parece sacar la consecuencia de manera adecuada.

Tomemos ahora el caso del Hipias Menor; se trata del primer diálogo que analiza Kutschera en su libro. Nos dice que "el peso de este diálogo está menos en el contenido filosófico que en la presentación de Sócrates en conversación" (I, 59). De ahí que lo pueda despachar en sólo siete páginas. Para Kutschera, el diálogo se mueve todo en el ámbito de la filosofía en su acepción primera, es decir, el saber elénctico que permite a Sócrates mostrarle a Hipias que no sabe lo que dice saber. Leer este diálogo de manera tan simple me parece un error. El Hipias Menor podrá ser un diálogo corto, pero presenta de manera comprimida una de las grandes preguntas que se hace Platón y a la que nunca responde de manera satisfactoria: si la virtud es un saber y si el saber concierne tanto a una cosa como a su contrario (un viejo topos griego), entonces la virtud es un saber del bien y del mal. Pero si eso es así, ¿cómo se podría evitar entonces que el virtuoso, el sabio, abuse de su saber y haga mal?

En el Hipias Menor, la discusión gira en torno a Odiseo, quien puede mentir con tanto éxito porque sabe mucho. En el Gorgias, la discusión gira en torno al orador, quien puede convencer a la multitud de cualquier cosa, buena o mala. Pero a final de cuentas, lo que vale para el orador vale para el filósofo. Todo saber se presta para usos múltiples; esto es parte del poder inherente al saber. La filosofía no escapa a esto ni puede escapar. Platón lo sabía; y jamás pudo construir una solución; jamás pudo salir del laberinto. Intentos filosóficos más recientes - por ejemplo, a partir de la reforma del platonismo que instaura Kant - son igualmente fallidos. Sin embargo, éste es un problema tan grave, tan serio, tan profundo de un cierto modo de filosofar que no verlo me parece una consecuencia de no tomar en serio la idea de filosofía como un saber en torno al 
"verdadero valor de las cosas", la tercera acepción teórica de la filosofía en la caracterización de Kutschera.

En este sentido diría que, si el lector se acerca a esta obra con el propósito de introducirse a la filosofía de Platón, se verá defraudado. La unidad filosófica que Kutschera pretende mostrar en Platón no se refiere sino a uno solo de los aspectos de la filosofía platónica, lo que podríamos llamar el aspecto lógico, refiriéndome con ello a la segunda acepción teórica de la filosofía, según Kutschera. Con respecto a este punto, yo diría que la contribución de Kutschera es sencillamente extraordinaria. La luz que arroja tanto sobre muchos diálogos individuales (el Carmides, el Eutidemo, el Fedón, la República, el Teeteto, el Sofista, el Timeo, el Filebo, pero sobre todo el Parménides) es tan intensa como la que arroja sobre lo que podemos saber de la "doctrina no escrita" de Platón.

He dicho antes que si el lector se acerca a esta obra con el propósito de introducirse a la filosofía de Platón, se verá defraudado. Hay una excepción: los dos capítulos introductorios son una buena síntesis en el nivel de quien inicia sus estudios (I, 13-58); no hay ciertamente nada original ni extraordinario en la presentación. Kutschera es un autor muy claro, pero no es un gran escritor ni alguien que pueda despertar mucho entusiasmo por su estilo. Pero presenta lo que se sabe y lo que se piensa en general (el consensus Platonicorum) de una manera sucinta y adecuada. Igualmente diría que la exposición final que Kutschera hace de su concepción integral de la filosofía de Platón es excelente, y lo primero que recomendaría al estudioso de Platón es que lo leyera (III, 173-235). No hay mejor orientación para después leer los análisis parciales que Kutschera hace de cada diálogo. Este capítulo sintético presenta la filosofía de Platón como si contuviera nueve elementos. Me parece que no violento a Kutschera cuando considero que esos elementos se pueden acomodar en tres grupos:

1. Las concepciones de Platón sobre las ideas (III, 175-194), la dialéctica (194-202) y los principios (202-206). 
2. Sus concepciones sobre la teología-teleología-teodicea (206$210)$, el dualismo (210-215) y la percepción en relación con el verdadero saber (215-222).

3. Sus concepciones sobre el bien vivir (222-226), el Estado $(227-230)$ y la filosofía misma (230-235).

El primer grupo es con mucho el mejor, aunque el segundo contiene ideas importantes. El tercer grupo es sobre el que Kutschera tiene menos que decir, pero también es el conjunto de ideas donde, en mi opinión, andaba más errado Platón. Ésta es probablemente la mejor explicación del carácter tan desigual del libro del que aquí me ocupo: Kutschera ha logrado dividir la filosofía de Platón en sus articulaciones naturales, para decirlo con el Fedro. Siendo así, la exposición no podría ser mucho mejor que su objeto.

Quisiera concluir con una brevísima indicación de en qué consiste la importantísima aportación de Kutschera a la interpretación del Parménides. En el análisis de la primera parte del diálogo sopla ya viento fresco, porque él con gran habilidad muestra lo endeble que son las críticas de Parménides a la teoría de las ideas desde un punto de vista lógico, y por ello aclara el verdadero sentido de esas críticas, a saber, la purificación elénctica del joven Sócrates (II, 168-182). Por ahí se ve lo errado que andaba Ryle al sugerir que la segunda parte del Parménides se habría añadido después. Para Kutschera, las dos partes van naturalmente juntas: debido a que Sócrates no entiende todavía la verdadera naturaleza de las ideas, sucumbe a las débiles críticas de Parménides, y por ello debe ejercitarse más en la diálectica.

Así se justifica el ejercicio lógico de la segunda parte. El tema de ese ejercicio no es en rigor el mismo que el de la primera, a saber, la participación de los objetos ordinarios en las ideas, sino un tema que se volverá el centro de las reflexiones lógicas más profundas del Platón maduro: la relación de las ideas entre sí (la koinonía ideón). Se trata de un tópico que había surgido aquí y allá en otros diálogos, pero sólo ahora se lo enfrenta en serio, tal vez porque hasta ese momento en el desarrollo del pensamiento 
de Platón dispone él de herramientas suficientes para afrontarlo. Esas herramientas pertenecen a una mereología (la lógica de todos y partes que Husserl vislumbró, pero que solamente Leśniewski supo desarrollar sistemáticamente). Ni qué decir tiene que Platón jamás dispuso de una mereología en el sentido moderno de la palabra; sin embargo, es posible mostrar que los argumentos de la segunda parte del Parménides dependen de ciertos principios lógicos que pertenecen a la mereología. Por supuesto que el análisis no es obvio, pero es posible mostrar que lo que en el diálogo se expresa con bastante oscuridad corresponde al uso de principios que podemos formular en el marco de un sistema formalizado que permite examinar la corrección de los argumentos parmenídeos.

En ese sentido podemos decir que los hallazgos lógicos de Platón no se limitan, como se había creído hasta Kutschera, a la idea de sistemas clasificatorios (la dihairesis que marca el inicio de la lógica silogística que desarrollaría Aristóteles) y al análisis de la oración en sujeto y predicado (esta innovación del Sofista que está en la base de la gramática griega y que, entre otras cosas, conducirá a la definición aristotélica de verdad). Platón sería también pionero en vislumbrar una lógica completamente distinta de la silogística e incluso del cálculo proposicional de los estoicos: si bien es posible representar los silogismos e incluso una parte de los razonamientos proposicionales en ella, se trata de un sistema independiente. Si Platón no lo desarrolló más allá de lo que podemos ver en el Parménides, eso se debe, sostiene Kutschera, a su creciente interés lógico en la predicación, que es un tema para el que la mereología no resulta muy apropiado (II, 200).

Cualquiera que haya luchado con ese texto tan enredado y confuso que es el Parménides le estará a Kutschera muy agradecido por la enorme luz que brinda su modo de analizarlo. Nuestro autor confiesa que quedan todavía muchas preguntas abiertas, y la mereología no parece ser bastante para responderlas; pero ya lo que hace es mucho. El lector interesado en estas cuestiones podrá introducirse en el asunto leyendo la breve descripción que hace Kutschera en el libro del que me ocupo 
aquí (II, 185-198); pero en último término deberá remitirse al tratamiento completo de la monografía antes mencionada (Platons "Parmenides").

En fin, aunque no se trata de un libro para principiantes, el filósofo formado sacará mucho provecho de su estudio. Si un alma caritativa se propone traducirlo, el trabajo será en un sentido fácil y en otro difícil; fácil, por cuanto Kutschera, como he dicho antes, escribe con claridad y desenvoltura; difícil, porque se requieren conocimientos tanto de filosofía antigua como de filosofía analítica, y tanto de lógica como un poco de matemáticas, física y teoría musical.

Platons Philosophie está muy bien impreso y encuadernado (rústica). La tipografía es cuidada, y no descubrí ningún error importante, lo que es admirable en un libro que contiene un número respetable de citas en griego, tanto como varios casos de notación lógico-matemática. Lo único que echo de menos es la falta de un índice analítico. En todo caso, para un libro tan bien hecho, el precio no me parece en absoluto excesivo. Por todo ello, no habrá que perder de vista las publicaciones de la editorial mentis (www.mentis.de), fundada hace relativamente poco tiempo, y dedicada principalmente a la filosofía de la mente y a la filosofía analítica en general.

\section{BIBLIOGRAFÍA}

Hadot, Pierre, Qu'est-ce que la philosophie antique?, Gallimard, París, 1995.

Platón, Timeo, trad. Francisco Lisi, Gredos, Madrid (Biblioteca Clásica, 160).

Steiner, Mark, The Applicability of Mathematics as a Philosophical Problem, Harvard University Press, Cambridge, Mass., 1998.

Von Kutschera, Franz, Die falsche Objektivität, De Gruyter, Berlín, 1993.

, Platons "Parmenides", De Gruyter, Berlín, 1995. 